\title{
PLANEJAMENTO ESTRATÉGICO E INFORMACIONAL DO DESENVOLVIMENTO DE UM NOVO PRODUTO: APLICATIVO DESTINADO ÀS EMPRESAS DO RAMO DE CONFEITARIAS
}

Rafael de Azevedo Palhares (rafaelpalhareseng@hotmail.com) - Ciências exatas, tecnológicas e humanas, UFERSA.

Darlly Dayanne da Silva dos Santos (darllydayanne@hotmail.com) - Ciências exatas, tecnológicas e humanas, UFERSA.

João Inácio de Oliveira da Silva (joao-inacio@live.com) - Ciências exatas, tecnológicas e humanas, UFERSA.

Samira Yusef Araújo de Falani (samira.yusef@ufersa.edu.br) - Ciências exatas, tecnológicas e humanas, UFERSA.

Camila Ingryd Vieira Trindade (camilaivtrindade@gmail.com) - Ciências exatas, tecnológicas e humanas, UFERSA.

\section{RESUMO}

O presente trabalho resulta na descrição do projeto de desenvolvimento de um novo aplicativo destinado ao setor de confeitarias. O aplicativo MyCake, visa fornecer um estreitamento relacional entre as empresas confeiteiras e seus clientes, possibilitando ao cliente a customização na montagem de bolos e visualização do seu pedido, bem como efetuar a compra onde estiverem. Foram aplicadas as ferramentas Brainstorming e o Método Score para auxiliar na tomada de decisão do produto a ser desenvolvido. Logo, foram realizadas pesquisas de mercado, por meio de questionário subjetivo e objetivo, coletando as informações dos potenciais clientes do aplicativo. A análise dos resultados se deu por meio do relacionamento entre o valor médio da avaliação estratégica e competitiva atribuída a cada requisito do aplicativo. Feito isto, concluiu-se que os itens prioritários para o desenvolvimento do produto são: Compatibilidade com mais de um sistema operacional; Fornecer claras informações sobre o produto e Usabilidade; Ressalta-se que todas essas especificações foram atendidas na etapa de montagem do protótipo. De acordo com os resultados, é possível concluir que o sucesso do aplicativo é consequiência do desenvolvimento do produto com foco nas especificações meta, visando $o$ atendimento da satisfação de todas as partes interessadas no produto.

Palavras-chave: Projeto de desenvolvimento de produto; Aplicativo MyCake; Setor de confeitarias. Área: Gestão do Processo de Desenvolvimento de Produtos 


\section{INTRODUÇÃO}

Para muitas empresas desenvolver novos produtos com maior rapidez, mais eficiência, e mais efetividade é a grande meta competitiva. Evidências provam que o design $\mathrm{e} o$ desenvolvimento efetivo de novos produtos têm um impacto significativo em custo, qualidade, satisfação do consumidor, e na vantagem competitiva. Através deste processo a empresa irá atingir novos mercados, introduzir inovações e aumentar seu potencial competitivo lançando novos produtos (DUTRA, 2011).

Uma das etapas que é indispensável para o desenvolvimento de produtos é a fase de identificar as oportunidades, na qual acontece uma geração de idéias, estas são analisadas, comparadas e escolhidas. Essa etapa acontece no planejamento estratégico do produto e foi exposto no trabalho com o auxilio do brainstorming (geração de idéias) e o método Score (analisar e escolher o produto para o desenvolvimento).

Dando continuidade para o desenvolvimento do produto é necessário também identificar os requisitos do mercado, e essa etapa ocorre no projeto informacional do produto, no qual foram aplicados questionários abertos (qualitativo) e fechados (quantitativos), com o intuito de obter as especificações dos futuros clientes do produto e assim encontrar o índice de importância da demanda corrigida pela qualidade (IDI), que será demonstrado no decorrer dos resultados.

Neste contexto, este artigo tem como objetivo apresentar a aplicação de uma metodologia, utilizada na disciplina Projeto e Desenvolvimento de Produto, para o de desenvolvimento de um novo produto. O produto proposto foi um aplicativo destinado as empresas do setor de confeitaria. Para isso, foi adotada uma metodologia composta pelas seguintes etapas: Planejamento estratégico e projeto informacional.

\section{MODELO DE REFERÊNCIA PARA O PROCESSO DE DESENVOLVIMENTO DE PRODUTOS - PDP}

De acordo com Souza e Queiroz (2015) é essencial que a realização de todas as etapas do desenvolvimento de produtos seja bem feitas, assim, o retrabalho não será necessários e a qualidade do produto é atendida de modo com as especificações do mercado sejam atendidas e o produto obtenha êxito.

Para Naka (2015), o processo de desenvolvimento de produtos compreende em um conjunto de atribuições que objetivam identificar expectativas de mercado, bem como restrições técnicas tecnológicas inerentes ao processo de desenvolvimento dos produtos em um bem de consumo disponível para venda. De acordo com Rozenfeld et al. (2010) o acompanhamento pós-lançamento do produto também é correspondente ao PDP de modo com que o planejamento de descontinuidade e todas as necessidades dos produtos sejam atendidos no decorrer do seu ciclo de vida.

O modelo intitulado como Modelo de Referência (MR) para o desenvolvimento de produtos é sugerido por Rozenfeld et al. (2006). O MR abrange desde o planejamento estratégico até a remoção do determinado produto no mercado. O Modelo de Referencia é seccionado em três fases macro, são elas: Pré-Desenvolvimento, Desenvolvimento e a ultima etapa sendo o pósdesenvolvimento.

O Pré-Desenvolvimento engloba as seqüenciais etapas: Planejamento Estratégico dos Produtos e Projeto do Produto. Inicialmente será definido o produto na qual deverá ser desenvolvido bem como a elaboração do planejamento do seu projeto. Na seguinte macro-fase Desenvolvimento englobam-se os seqüenciais projetos: Projeto Informacional, Projeto Conceitual, Projeto Detalhado, Preparação da Produção e Lançamento do Produto. A última 
marco-fase do modelo é o Pós-Desenvolvimento, esta incorpora as seguintes etapas: Acompanhamento do Produto e descontinuidade no mercado, conforme será revelado na Figura 1.

Figura 1. Processo de desenvolvimento de produto. Fonte: Rozenfeld et al. (2006)

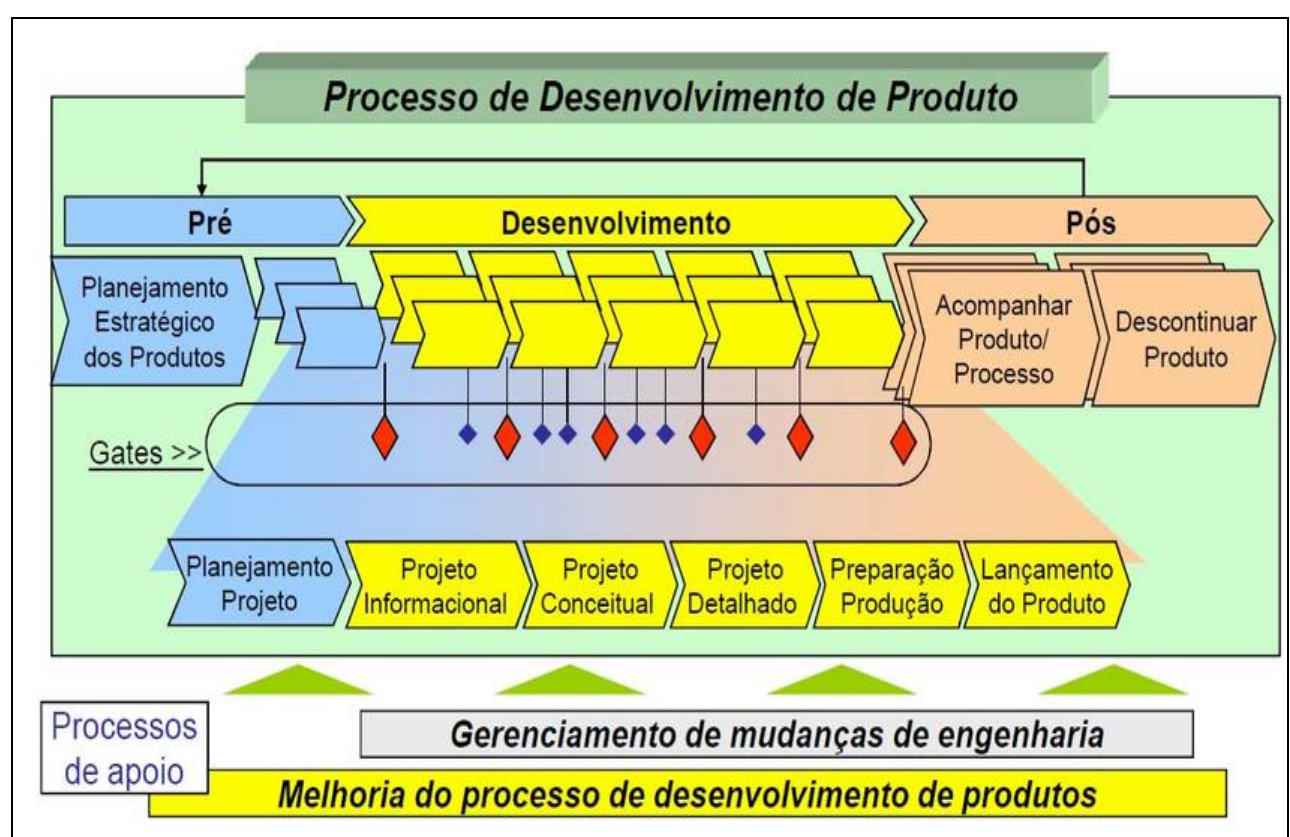

As etapas tratadas neste artigo são o Planejamento Estratégico de Produtos e o Projeto Informacional das respectivas macro-fases, Pré desenvolvimento e Desenvolvimento. O progresso dessas etapas é de fundamental importância, pois determinam qual produto será desenvolvido e as suas principais características para que satisfaçam os requisitos dos potenciais clientes, sendo esses fatores de sucesso para o lançamento de novos produtos.

\subsection{Planejamento estratégico do produto}

De acordo com modelo de referencia do autor Rozenfeld et al. (2010) esta é a etapa inicial para o pré-desenvolvimento do produto, seqüencialmente realiza-se a etapa de planejamento do projeto do produto. Dentre as atividades pertinentes ao planejamento estratégico do produto podem ser destacadas: Identificação dos interesses estratégicos da organização, disponibilidade dos recursos, bem como deve ser elaborado pesquisas de mercado, na qual o ambiente externo (concorrentes e clientes) sejam analisados e inseridos no planejamento de implementação do produto.

Ainda de acordo com Rozenfeld et al. (2010) o intuito do Planejamento Estratégico de Produtos consiste em recapitular o Plano Estratégico de negócios (PEN), de modo com que as informações do mercado e tecnologia sejam firmadas. É também correspondente a esta etapa, o diagnóstico da viabilidade do portfólio estabelecido, proposição de mudanças e escolha de um dos produtos do portfólio para que o planejamento seja iniciado.

Neste trabalho as ferramentas adotadas são: brainstorming, na qual Ormandy (2012) classifica como um método usado para encontrar um determinado assunto para certa hipótese. Como também o método Score, que se propõe na avaliação do portfólio, sua metodologia aplica-se na atribuição de notas alocadas a critérios definidos pelos desenvolvedores de modo a concluir que o produto detentor da maior pontuação seja indicado como o produto a ser desenvolvido. 


\subsection{Projeto Informacional}

Rosenfeld et al. (2010) declara que no projeto informacional deve estar contido todos os elementos requisitados em relação ao produto. Informações no tocante as especificações e características do produto devem ser claramente definidas de modo com que as expectativas dos clientes e da organização criadora venham ser almejadas. É uma das fases decisivas para o êxito do produto no mercado.

Ainda de acordo com Rosenfeld et al. (2010) o intuito deste projeto consiste na revisão e atualização do escopo, detalhamento do ciclo de vida do produto e a identificação das características requisitadas, logo, as especificações meta devem ser definidas e a viabilidade econômica deve ser monitorada.

Nesta pesquisa, a coleta dos requisitos dos produtos e conversão em especificações do produto foi obtida a partir da aplicação de dois tipos de questionários, sendo eles: Questionário qualitativo e quantitativo de modo com que a análise do índice de importância corrigido pela qualidade pudesse ser observada e estes requisitos viessem ser revelados em um ranking de importância.

\section{PROCEDIMENTOS METODOLÓGICOS}

O presente trabalho trata-se de um recorte do Projeto de Desenvolvimento de um Novo Produto apresentado em uma disciplina do curso de Engenharia de Produção. Primeiramente foi ocorreu a elaboração no Planejamento Estratégico do produto, em que foram aplicadas as ferramentas brainstorming e o método Score para se definir o produto a ser desenvolvido.

Foi realizada ainda uma consulta ao site do Instituto Nacional de Propriedade Intelectual (INPI) de modo a identificar se aquele produto a ser desenvolvido não estava registrado, verificando se o produto era inédito no mercado e não infringia a legislação de propriedade intelectual.

No projeto informacional, foi determinado o público-alvo, e elaborados dois questionários (qualitativo e quantitativo) a fim de identificar os requisitos desejados pelos clientes e a sua importância, sendo no questionário qualitativo expressados de forma subjetiva o que se espera com o produto, e no quantitativo identificada a importância de cada característica citada no primeiro questionário. A aplicação dos questionários se deu por meio do Google Formulário na qual a pesquisa obteve 47 entrevistados, dentre empresas de confeitarias e clientes de empresas deste seguimento.

Obtidas as informações do questionário quantitativo, foi possível identificar o índice de importância corrigido pela qualidade, que confronta a importância atribuída pelos potenciais clientes, pelos envolvidos no desenvolvimento do produto e pelo desempenho do produto em relação a um produto similar concorrente, resultando em um ranking de priorização dos requisitos.

Diante das características mais importantes elencadas pelos interessados no projeto, foi possível elaborar as interfaces preliminares do aplicativo por meio de um software de desenho da Microsoft. 


\section{PROCESSO DE DESENVOLVIMENTO DO APLICATIVO MYCAKE}

\subsection{Aplicação do Brainstorming}

Os próprios autores estabeleceram diversas concepções e ideias de novos produtos que possivelmente viessem a ser desenvolvidos. Em seqüência, uma filtragem considerando a viabilidade técnica, econômica, tecnológica, bem como as ideais que obteriam maior retorno para a sociedade e desenvolvedores foi aplicada.

Para concretizar a escolha do produto foi realizada uma geração de opções e alternativas de ideias foram escolhidas as opções mais interessantes para o interesse da equipe de trabalho, sendo elas: Software para pedidos de bolo personalizados, Software para gerenciamento de salas Software do ônibus da Universidade Federal Rural do Semi-Árido (UFERSA), Software para planejamento de horário e Software para pedir moto taxi.

Após todas as ideias apresentadas foi escolhida as mais viáveis e mais aceitáveis no mercado, analisando também a existência desses produtos, ou seja, eliminação de produtos já existentes. Sendo assim, após o refinamento, ficaram os produtos: Software para pedidos de bolo personalizados, Software do ônibus da UFERSA e Software para pedir moto taxi.

\subsection{Utilização do método Score}

Após a identificação dos possíveis produtos a serem desenvolvidos, observou-se a necessidade de utilização de uma ferramenta para apoiar a decisão de qual produto seria desenvolvido. Assim optou-se pelo Método Score, que auxilia a tomada de decisão na gestão de portfólios de projetos, partindo da identificação de critérios, pesos e atribuição de notas. Onde foram classificadas três alternativas de novo produto.

A primeira etapa foi determinar os critérios que se encaixam nas alternativas citadas anteriormente: Lucratividade, Tempo de lançamento (curto), Interesse do consumidor, Viabilidade técnica e Custos. Foi necessário estabelecer ainda os pesos de cada critério, onde cada critério recebia um peso que variava de 0 a 2 . A avaliação era classificada como: 0 , quando menos importante, 1 quando de igual importância e 2 quando mais importante, que corresponde ao modelo baseado em notas (Score). As resoluções dos critérios atribuídos aos pesos estão representadas na Figura 2.

Figura 2. Pesos atribuídos aos critérios.

\begin{tabular}{ccccccc}
\hline & Lucratividade & Tempo & $\begin{array}{c}\text { Interesse do } \\
\text { consumidor }\end{array}$ & $\begin{array}{c}\text { Viabilidade } \\
\text { técnica }\end{array}$ & Custos & Peso \\
\hline $\begin{array}{c}\text { Lucratividade } \\
\text { Tempo de lançamento } \\
\text { (curto) }\end{array}$ & - & 2 & 1 & 0 & 2 & 5 \\
Interesse do consumidor & 1 & - & 0 & 1 & 2 & 3 \\
Viabilidade técnica & 2 & 1 & 1 & 1 & 2 & 6 \\
\hline Custos & 0 & 0 & 0 & 0 & - & 0 \\
\hline
\end{tabular}

Logo após essa etapa, foram atribuídas as notas para cada possível produto resultado do brainstorming, com o intuito de fazer a média e saber qual produto seria o escolhido para o desenvolvimento. Assim, a equipe avaliou em notas de 1 a 5 os critérios em relação aos produtos, sendo 1 seria o pior desempenho no critério e 5 o melhor desempenho. Após a 
obtenção das medias, foi realizado o calculo da média ponderada das alternativas, baseado nos pesos atribuídos anteriormente, que está apresentada na Figura 3.

Figura 3. Médias ponderadas dos possíveis produtos.

\begin{tabular}{|c|c|}
\hline & Média ponderada \\
\hline Software de bolo & 3,93 \\
\hline Software de moto taxi & 3,87 \\
\hline Software de ônibus & 3,5 \\
\hline
\end{tabular}

Dessa forma, de acordo com os resultados obtidos, o produto com maior nota foi o software do bolo, consequentemente, foi o escolhido para o desenvolvimento do produto.

\subsection{Questionário Qualitativo e Quantitativo}

Foi elaborado um questionário com perguntas subjetivas destinado aos dois segmentos de clientes: empresa e usuários (consumidores), com o objetivo de especificar com clareza as necessidades dos clientes.

Para as empresas foram explicitados os seguintes questionamentos: Quais características que o aplicativo deve apresentar de modo com que o resultado esperado com a utilização do aplicativo seja o melhor; Se os clientes já utilizam ou conhece algum tipo de ferramenta voltado para essa área de confeitaria; Sistema operacional desejável, Quais funções devem conter no aplicativo e qual a disposição financeira da empresa para pagar pela cessão dos direitos do aplicativo. No questionário voltado para os usuários finais foram abordados os seguintes pontos; A escolha das características que o aplicativo deve apresentar melhor resultado esperado com a utilização do aplicativo; Se os clientes já utilizam ou conhece algum tipo de ferramenta voltado para essa área de confeitaria; Tipos de sistemas operacionais desejáveis e como gostaria deveria ser a interface do aplicativo.

A partir das respostas obtidas no primeiro questionário, pôde-se elaborar a árvore da demanda da qualidade, que consiste em classificar os requisitos respondidos em itens primários e secundários, possibilitando a criação do questionário quantitativo, neste, os usuários atribuíram notas de importância para as características identificadas. As Figuras 4 e 5 expõem as características definidas como níveis primários e secundários dos clientes empresa e usuários finais. 
Figura 4. Árvore da Qualidade demandada - Empresas e Autônomos.

\begin{tabular}{|c|c|}
\hline Nível primário & Nível secundário \\
\hline Características & Usabilidade da ferramenta \\
& $\begin{array}{c}\text { Agilidade no processamento do pedido } \\
\text { Visualização do pedido antes da compra } \\
\text { Pequena ocupação de memória } \\
\text { Clareza nas informações do produto }\end{array}$ \\
\hline Resultados esperados & $\begin{array}{c}\text { Satisfação do cliente } \\
\text { Retomo financeiro }\end{array}$ \\
& Facilitar a comunicação com o cliente \\
\hline Funções & Exibição de imagens do produto \\
& Variadas opções de montagem do \\
& pedido \\
& Anúncio de promoções \\
& Atendimento ao cliente \\
& Pós-venda (Avaliação) \\
\hline Sistema operacional & Android \\
iOS
\end{tabular}

Figura 5. Árvore da Qualidade demandada - Usuários finais.

\begin{tabular}{|c|c|}
\hline Nível primário & Nível secundário \\
\hline Benefícios & Rapidez no pedido \\
& Acessibilidade aos pedidos \\
Informações sobre o produto \\
Comunicação
\end{tabular}

A partir dos resultados obtidos com a aplicação do questionário quantitativo, foi possível identificar o índice de importância corrigido da qualidade demandada (IDi*). Cada requisito é graduado de acordo com sua importância, ou seja, a importância atribuída pelos respondentes é confrontada com a importância atribuída pela equipe desenvolvedora, de modo com que cada requisito pudesse ser analisado. A obtenção do cálculo do IDi* baseou-se na equação 1 . A própria equipe desenvolvedora realizou a avaliação competitiva (Mi) e estratégica (Ei) dos requisitos.

$$
\mathrm{IDi}^{*}=\mathrm{IDi} \times \sqrt{\mathrm{Ei}^{i}} \times \sqrt{\mathrm{Mi}} \text { (Equação 1) }
$$

Sendo:

- IDi* = Índice de importância corrigido da qualidade demandada;

- IDi = Importância dos itens da qualidade demandada (Peso relativo);

- $\quad \mathrm{Ei}$ = Avaliação estratégica dos itens da qualidade demandada;

- $\mathrm{Mi}=$ Avaliação competitiva dos itens da qualidade demandada.

Foi necessário o tratamento dos dados para cada um dos requisitos, encontrando a média de importância atribuída pelos entrevistados, assim foi possível identificar o peso relativo de 
cada item. As notas (Mi) e (Ei) foram decididas pelos próprios desenvolvedores a partir das equivalências expostas na Tabela 1 .

Tabela 1. Notas de Avaliação competitiva e estratégica.

\begin{tabular}{|c|c|}
\hline $\begin{array}{c}\text { Equivalência das notas correspondentes a } \\
\text { avaliação competitiva (Mi) }\end{array}$ & $\begin{array}{c}\text { Equivalência das notas correspondentes a } \\
\text { avaliação estratégica (Ei) }\end{array}$ \\
\hline 0,5 - Acima da concorrência; & 0,5 - Importância pequena; \\
\hline 1,0 - Similar a concorrência; & 1,0 - Importância média; \\
\hline 1,5 - Abaixo da concorrência; & 1,5 - Importância grande; \\
\hline 2,0 - Muito abaixo da concorrência. & 2,0 - Importância muito grande. \\
\hline
\end{tabular}

A partir do resultado da equação para cada requisito, foi possível realizar um ranking referente ao grau de importância dos itens e construção do gráfico que expressa os principais itens de qualidade a serem atendidos no aplicativo MyCake. A figura 6 revela o resultado referente ao $\mathrm{IDi}^{*}$ de cada um dos requisitos identificados pelos clientes empresa e usuários finais.

Figura 6. Nível de Importância - Priorização dos itens.

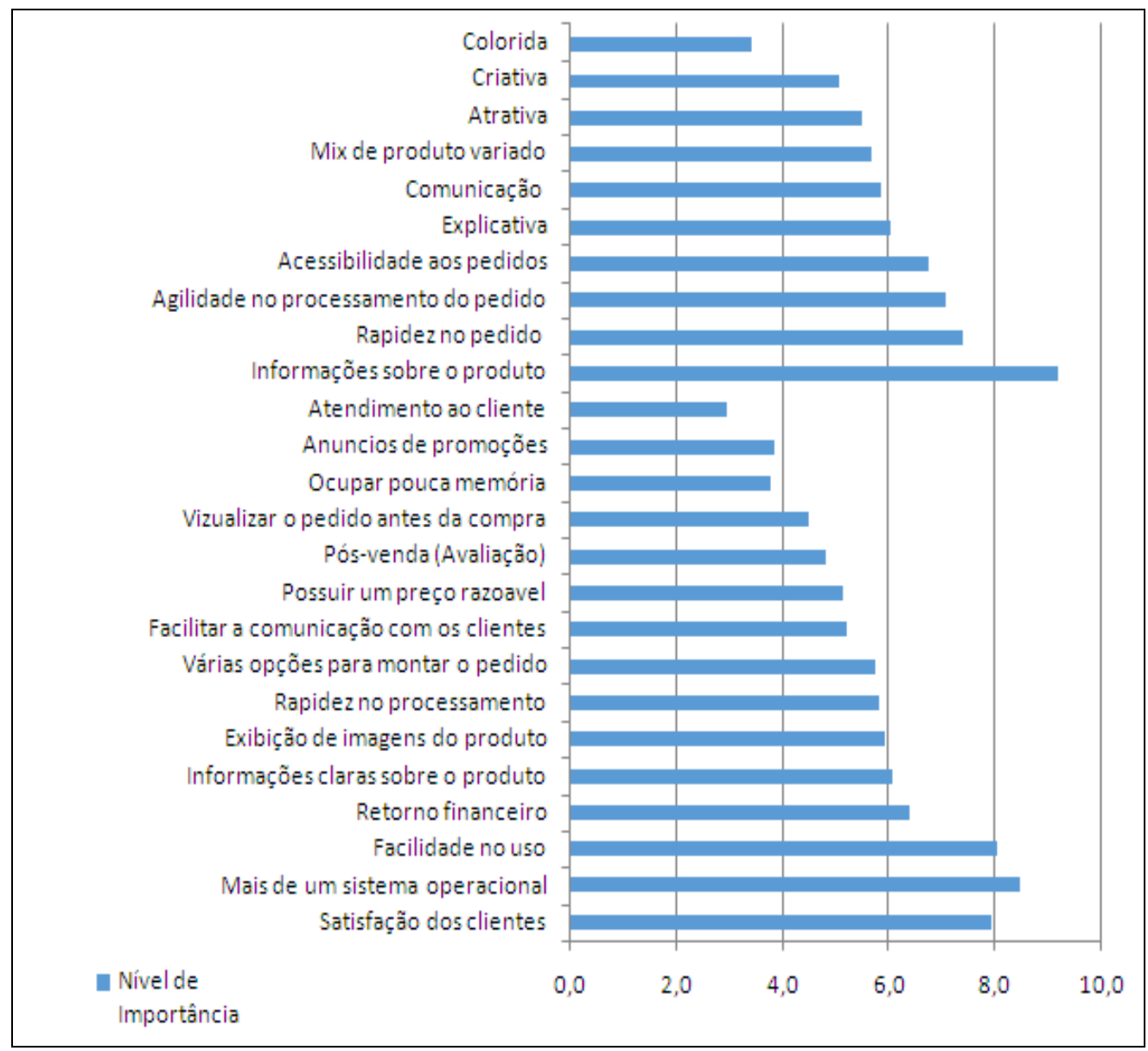


É possível identificar que os requisitos prioritários para o desenvolvimento do aplicativo MyCake foram: informações claras sobre o produto ofertado, tais como, ilustrações, preço, sabor, cores e etc.; abrangência em mais de um sistema operacional; e a facilidade de uso.

\section{APLICATIVO MYCAKE}

Com base nos requisitos e especificações que foram identificadas nas etapas anteriores, foram definidas características para o produto e então elaboradas as interfaces do mesmo. Para melhor entendimento do funcionamento do aplicativo, foi feito um fluxograma para um tipo de pedido, e dois tipos de usuários: Cliente final e empresa.

No fluxo, tem-se o passo a passo para a realização de um pedido no aplicativo, onde o usuário tem a opção de se cadastrar como empresa, registrando produtos e atendendo clientes por meio do chat do aplicativo, ou como cliente, cadastrando suas informações, decidindo sobre a empresa que pretende visualizar os produtos (prontos ou personalizados) a serem escolhidos, definindo a forma de pagamento e finalizando a compra, além de uma interface destinada a comunicação (chat) entre empresa e cliente final. O fluxo é demonstrado na figura 5.

Figura 5. Fluxograma das interfaces do aplicativo MyCake

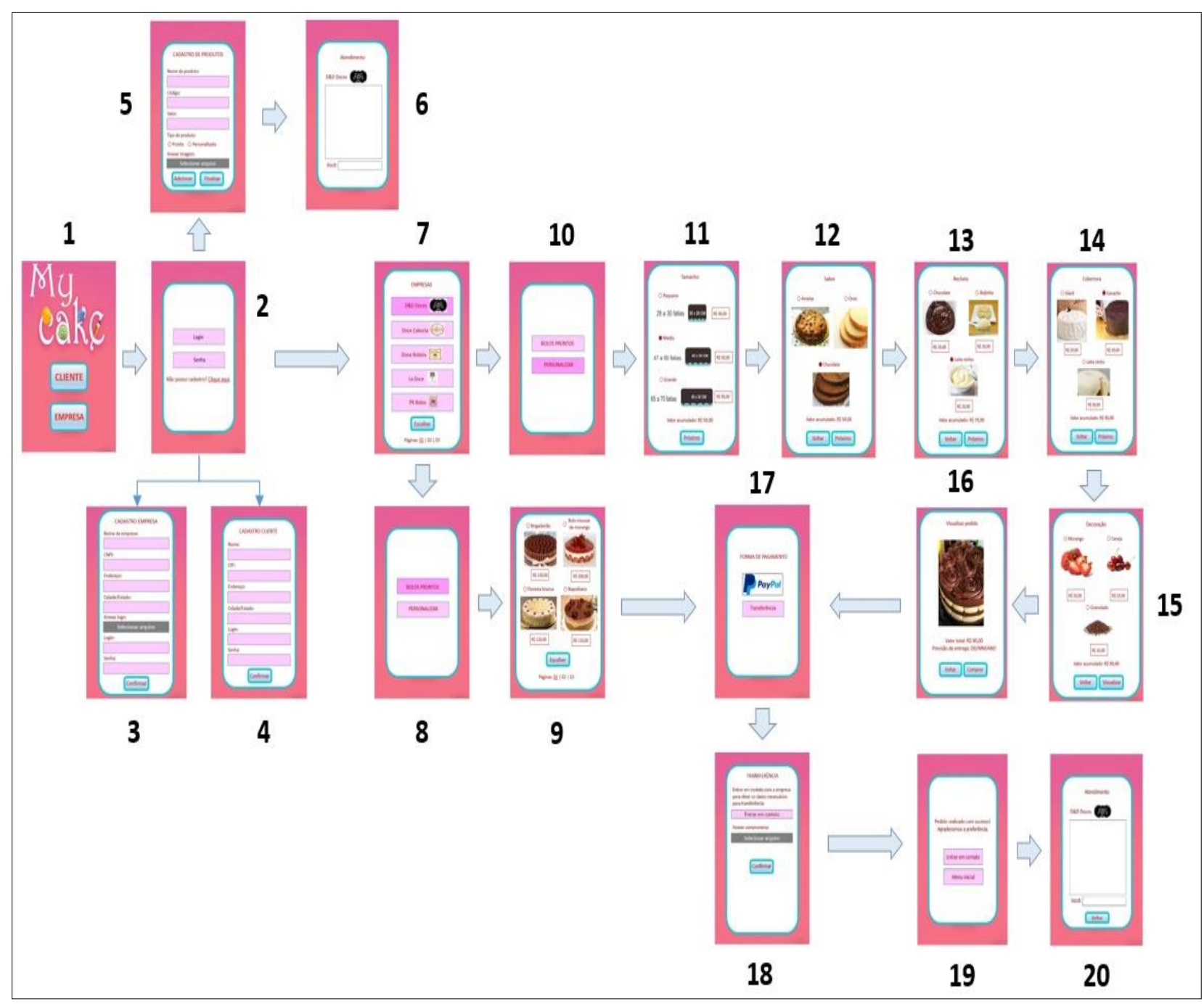


Vale salientar que cada item das especificações metas foram atendidos, como pode-se observar entre as interfaces 7 e 16, que reproduzem informações claras sobre o produto, apresentado ilustrações reais disponibilizadas pelas empresas, sempre informando o preço unitário para cada opção. Além disso, o aplicativo será compatível com dois dos principais sistemas operacionais: iOs e Android. Disponibilizando interfaces virtuais simples e interativas, similar a muitos aplicativos que já existem no mercado, com um linguajar compreensível e funções autoexplicativas, utilizando de botões e menus que o usuário já tem costume de lidar.

\section{CONSIDERAÇÕES FINAIS}

O desenvolvimento de produtos é uma atividade importante e ariscada, que visa atender as necessidades dos clientes, transformando as informações do mercado em requisitos do produto, o que se apresenta como um processo crítico para a competitividade das empresas.

O presente trabalho consistiu no desenvolvimento de um aplicativo destinado ao setor de confeitarias de bolos, visando a diversificação do atendimento e melhor interação das empresas com os seus clientes. $\mathrm{O}$ aplicativo MyCake poderá ser utilizado pelas empresas que pretendem divulgar os seus produtos e oferecer a opção de personalização destes, assim como pelos clientes que buscam agilidade no pedido, mantendo a qualidade e diversidade dos produtos, além da segurança na compra.

Para isso, foram aplicadas ferramentas como brainstorming, método Score, questionários qualitativos e quantitativos, a fim de identificar o produto que mais se adeque as necessidades do mercado e recolhendo informações que foram transformadas em especificações meta para o produto, e consequentemente promovesse a satisfação dos clientes.

Visando a continuidade do processo de desenvolvimento do aplicativo MyCake, este artigo abre espaço para a realização de trabalhos futuros sobre o tema, que demonstrem a importância da criação de novos produtos e como o PDP pode auxiliar nesse processo.

\section{REFERÊNCIAS}

DUTRA, Adriana Conceição; LOPES, Claudimar de Souza; GARCIA, Mere Helen. Marketing de novos produtos lançamento e inovação. Revista Científica do Unisalesiano Lins - SP, ano 2, n.5, Edição Especial, outubro 2011.

NAKA, Bruno Afonso Hayaki. Modelo de desenvolvimento de produtos para uma empresa de artefatos de alumínio. 2015. 61 f. TCC (Graduação) - Curso de Engenharia Mecânica, Universidade Tecnológica Federal do Paraná, Pato Branco, 2015.

ORMANDY, L. Brainstorming for a topic. Author's Edition. [S.I]: [s.n], 2012.

Rozenfeld, H.; Forcellini, F. A.; Amaral, D. C.; Toledo, J. C.; Silva, S. L. d.; Allipradini, D. H. \& Scalice, R. K. (2006), Gestão de Desenvolvimento de Produtos, Saraiva.

ROZENFELD, Henrique; FORCELLINI, Fernando Antônio; AMARAL, Daniel Capaldo. Gestão de desenvolvimento de produtos: uma abordagem para a melhoria de processos. São Paulo: Saraiva, 2010.

SOUZA, Priscila Aparecida Santos de; QUEIROZ, Stefânia Aparecida Belute. Um olhar para a qualidade no processo de desenvolvimento de produtos de uma empresa de médio porte fabricante de Lingerie. Revista de Iniciação Científica da Libertas, São Sebastião do Paraíso, v. 5, n. 1, p.1-18, dez. 2015. 